Journal of Applied AnALysis

Vol. 6, No. 2 (2000), pp. 187-211

\title{
THE EXISTENCE OF MOMENTS OF SOLUTIONS TO TRANSPORT EQUATIONS WITH INELASTIC SCATTERING AND THEIR APPLICATION IN THE ASYMPTOTIC ANALYSIS
}

\author{
J. BANASIAK
}

Received October 10, 1999 and, in revised form, March 28, 2000

\begin{abstract}
In this paper we prove the existence of all moments of the solutions to the time-dependent spatially homogeneous transport equation describing elastic and inelastic scattering of particles. The proof uses the theory of resolvent positive operators and Desch's perturbation theorem. As an application we carry out the asymptotic analysis of the full transport equation with dominant elastic scattering and, using the results of the first part of the paper, we show that its solution can be approximated in the $L_{1}$-norm by the solution of the limit equation obtained by formal asymptotic expansion.
\end{abstract}

\section{Introduction}

Several recent years witnessed a renewed interest in transport equations describing both elastic and inelastic scattering of particles. Such models are relevant to the semiconductor processes (see e.g. [10, 18, 19, 20] and

1991 Mathematics Subject Classification. 34G10, 82C40, 47D06, 35 Q35.

Key words and phrases. Evolution equations, positive semigroups, inelastic scattering, singular perturbations, hydrodynamic limit.

This work was supported by grants from the National Research Foundation of South Africa and the University of Natal Research Fund.

ISSN 1425-6908 CHeldermann Verlag. 
many others), and also to various electron and neutron transport processes in gases and solids (see e.g. [17] and references therein, also [9, 3]). The term "inelastic scattering" refers here to the fact that the moving particles can exchange quantized portions of energy with the background - a more detailed description of the physical situation leading to such models is given in Section 2.

Elastic and inelastic scattering in a given model can occur with different intensity or on different time scales. Mathematically this is accounted for by the introduction of dimensionless parameters multiplying appropriate terms of the equation, and indicating their relative magnitudes. In some cases certain processes dominate the others which justifies analyzing the behaviour of the equation when relevant parameters tend to infinity, and studying the possible limit cases.

Such an analysis is also important from the computational point of view as the limit equations are usually much simpler than the original ones. Thus, if we know that the difference between the solutions to the limit equation and to the original equation is small, we can use the former, obtained with much less computational effort, as an approximation to the latter.

An exhaustive discussion of various asymptotic limits for semiconductor equations can be found in [10]. The aim of this paper, which is a part of a wider collection of works $([4,8,9,12,14])$, is to investigate possible asymptotic limits for another class of transport equations. These equations contain no external field, which makes them easier than semiconductor equations but, contrary to the latter, contain an unbounded operator responsible for the inelastic scattering, which makes the rigorous mathematical analysis quite involved.

The main aim of this paper is to prove that the solutions of time-dependent spatially homogeneous equation with unbounded inelastic scattering operator have all the moments. Precisely speaking, we prove that this operator generates a positive semigroup in appropriately weighted $L_{1}$ spaces. This is done by careful estimations of the resolvent of the inelastic scattering operator and an application of Desch's theorem for resolvent positive perturbations of positive semigroups (see e.g. [22]).

An application of this result is demonstrated in the last section of the paper. We use it to prove that the formal asymptotic expansion yields the correct approximation of the solution of the transport equation, and to provide a sharp estimate of the error of this approximation in physically natural $L_{1}$ space. 


\section{Description of model}

We consider a gas of test particles having mass $m$ propagating through a host medium of particles having mass $M$, and undergoing two types of collisions with them: elastic and inelastic ones.

We assume that the host medium is at thermodynamical equilibrium with temperature $T$ and we consider the Lorentz gas limit $(m / M<<1$, in other words, the test particles collide with something like a rigid net they can be deflected (elastic collisions) or exchange quanta of energy with the background (inelastic collisions), but the classical exchange of kinetic energy is ruled out). For technical reasons we assume that the scattering is isotropic.

Furthermore, the background particles can occur in two energy states: a ground level and an excited level, spaced by an energy gap denoted by $\Delta E$ (which throughout the paper will be normalized to one). The number densities of the particles in the ground and in the excited states are assumed to be constant; we denote them by $n_{1}$ and $n_{2}$, respectively. They are related through the Boltzmann factor $a:=n_{2} / n_{1}=e^{-\Delta E / k T}$, where $k$ is the Boltzmann constant.

We are concerned with two scattering operators: inelastic and elastic ones. The elastic scattering operator is given by

$$
\left(C^{e} f\right)(\boldsymbol{v})=-f(\boldsymbol{v}) \lambda(v)+\frac{1}{4 \pi} \lambda(v) \int_{S^{2}} f\left(v \boldsymbol{\omega}^{\prime}\right) d \boldsymbol{\omega}^{\prime},
$$

where $f$ is the density of the test particles, $S^{2}$ denotes the unit sphere in $\mathbb{R}_{v}^{3}, \boldsymbol{v}=v \boldsymbol{\omega}$ with $v \in\left[0, \infty\left[\right.\right.$ and $\boldsymbol{\omega} \in S^{2}$. Since we will be working with the Maxwell molecules, we assume that the elastic scattering cross-section $\lambda$ is a measurable function satisfying:

$$
\lambda_{\min } \leq \lambda(v) \leq \lambda_{\max }
$$

for almost all $v \in\left[0, \infty\left[\right.\right.$ and some $0<\lambda_{\min } \leq \lambda_{\max }<+\infty$.

The inelastic scattering operator is given by

$$
\begin{aligned}
\left(C^{i} f\right)(\boldsymbol{v})= & -f(\boldsymbol{v})\left(\frac{n_{2} v_{+}}{v} \nu\left(v_{+}\right)+n_{1} H\left(v^{2}-1\right) \nu(v)\right) \\
& +\frac{n_{1}}{4 \pi v} \int_{S^{2}} v_{+} \nu\left(v_{+}\right) f\left(v_{+} \boldsymbol{\omega}^{\prime}\right) d \boldsymbol{\omega}^{\prime} \\
& +\frac{n_{2}}{4 \pi} \int_{S^{2}} H\left(v^{2}-1\right) \nu(v) f\left(v_{-} \boldsymbol{\omega}^{\prime}\right) d \boldsymbol{\omega}^{\prime} .
\end{aligned}
$$

where $H$ denotes the Heavyside function, and $v_{ \pm}=\sqrt{v^{2} \pm 1}$. 
As before, the Maxwell molecule hypothesis requires that the inelastic scattering cross-section $\nu$ satisfies

$$
0<\nu_{\min } \leq \nu(v) \leq \nu_{\max }<+\infty \text { for } v \in[1, \infty[.
$$

Note that due to the appearance of the Heavyside function and the shift of the velocity, there is no need to specify the behaviour of $\nu$ for $v \in[0,1[$.

This model is a simplified version of the general inelastic scattering model developed in $[17,11,3]$ where also a more detailed explanation of the physics of the problem is provided.

We note ( e.g. $[3,5])$ that under the adopted assumptions the operator $C^{e}$ is bounded in $L_{1}\left(\mathbb{R}^{3}\right)$. In contrast, the operator $C^{i}$ is not bounded in this space. However, both operators are conservative, that is, the integrals over $\mathbb{R}^{3}$ of both $C^{i} f$ ( $f$ in the domain of $C^{i}$ ) and $C^{e} f$ are equal to zero.

It greatly simplifies the notation if one changes the velocity to the normalized kinetic energy according to the formula $v^{2}=\xi$. In what follows, with some abuse of notation, we shall write $f(\xi \pm 1, \boldsymbol{\omega})$ instead of $f\left(v_{ \pm} \boldsymbol{\omega}\right)$.

The physically relevant space for our considerations is the space

$$
X=L_{1}\left(\mathbb{R}^{3}\right),
$$

as the integral of the density $f$ over $\mathbb{R}^{3}$ gives the total number of particles. It is useful to note that when we pass to the $\xi$ variable, then the measure $d \boldsymbol{v}=v^{2} d v d \boldsymbol{\omega}$ changes into $d \boldsymbol{\xi} / 2$, where $d \boldsymbol{\xi}=\sqrt{\xi} d \xi d \boldsymbol{\omega}$.

Since we will be working quite a lot with isotropic ( $\boldsymbol{\omega}$ independent) functions, it is worthwhile to note that for such functions we have $X=$ $L_{1}\left(\left[0, \infty\left[, v^{2} d v\right)=L_{1}([0, \infty[, \sqrt{\xi} d \xi)\right.\right.$.

We note that the inelastic scattering operator splits in a natural way into two operators: the down-scattering operator $C_{-}^{i}$, given by

$$
\begin{aligned}
\left(C_{-}^{i} f\right)(\xi, \boldsymbol{\omega})= & -\alpha_{-}(\xi) f(\xi, \boldsymbol{\omega})+\left(K_{-}^{i} f\right)(\xi) \\
= & -n_{1} H(\xi-1) \nu(\xi) f(\xi, \boldsymbol{\omega}) \\
& +\frac{n_{1}}{4 \pi} \sqrt{\frac{\xi+1}{\xi}} \nu(\xi+1) \int_{S^{2}} f(\xi+1, \boldsymbol{\omega}) d \boldsymbol{\omega}
\end{aligned}
$$

and the up-scattering operator, defined as

$$
\begin{aligned}
\left(C_{+}^{i} f\right)(\xi, \boldsymbol{\omega})= & -\alpha_{+}(\xi) f(\xi, \boldsymbol{\omega})+\left(K_{+}^{i} f\right)(\xi) \\
= & -n_{2} \sqrt{\frac{\xi+1}{\xi}} \nu(\xi+1) f(\xi, \boldsymbol{\omega}) \\
& +\frac{n_{2}}{4 \pi} H(\xi-1) \nu(\xi) \int_{S^{2}} f\left(\xi-1, \boldsymbol{\omega}^{\prime}\right) d \boldsymbol{\omega}^{\prime},
\end{aligned}
$$


where the definitions of the functions $\alpha_{ \pm}$and the operators $K_{ \pm}^{i}$ follow from the above equations in a self-explanatory way.

From the physical point of view the down-scattering operator gives the balance of particles which lose energy in the interaction, whereas the upscattering balances those particle which gain energy in the interaction. It follows [3] that $C_{-}^{i}$ is a bounded operator in $X$, whereas $C_{+}^{i}$ is unbounded in this setting.

We consider $C_{+}^{i}$ (and thus $C^{i}$ and $C^{i}+C^{e}$ ) on the domain $D(C)=$ $L_{1}\left(\mathbb{R}^{3},\left(1+v^{-1}\right) d \boldsymbol{v}\right)$. It is clear that $D(C)$ is dense in $X$ as if $f \in X$, then $f_{\varepsilon}=f\left(1-\chi_{[-\varepsilon, \varepsilon]}\right) \in D(C)$, where $\chi_{A}$ is the characteristic function of $A$, and $f_{\varepsilon} \rightarrow f$ as $\varepsilon \rightarrow 0^{+}$in $X$.

Note that for isotropic functions we have $D(C)=L_{1}([0, \infty[,(\sqrt{\xi}+1) d \xi)$.

We shall consider the time-dependent spatially homogeneous Boltzmann equation for elastic and inelastic scattering

$$
\partial_{t} f=C^{i} f+C^{e} f
$$

with the initial condition

$$
f(\boldsymbol{v}, 0)=\stackrel{\circ}{f}(\boldsymbol{v}) .
$$

The solvability of (2.6) in $X$ follows from more general results in $[3,7]$ where also various effects of the spatial inhomogeneity were taken into account. In this paper, however, we are concerned with the existence of moments of the solution to $(2.6):(\boldsymbol{v}, t) \rightarrow v^{k} f(t, \boldsymbol{v}), k=1,2 \ldots$ or, in other words, with the existence of solutions of (2.6) in the weighted spaces

$$
X_{k}=L_{1}\left(\mathbb{R}^{3},\left(1+v^{k}\right) d \boldsymbol{v}\right)=L_{1}\left(\mathbb{R}^{3},\left(1+\xi^{k / 2}\right) d \boldsymbol{\xi}\right) .
$$

Remark 2.1. Before we proceed any further, we note that it seems that this problem cannot be dealt with by the methods developed in $[3,7]$, as the the latter heavily depended on the conservativeness of the collision operators in $X$. In $X_{k}$, however, the conservativeness is lost. To show this, let us first note that

$$
\xi^{k / 2}\left(C^{e} f\right)(\xi, \boldsymbol{\omega})=C^{e}\left(\xi^{k / 2} f\right)(\xi, \boldsymbol{\omega}) .
$$

On the other hand, we have

$$
\begin{aligned}
\left(\xi^{k / 2} C^{i} f\right)(\xi, \boldsymbol{\omega}) & =-\left(\alpha_{-}(\xi)+\alpha_{+}(\xi)\right) \xi^{k / 2} f(\xi, \boldsymbol{\omega}) \\
& +\frac{n_{2} H(\xi-1) \nu(\xi)}{4 \pi} \frac{\xi^{k / 2}}{(\xi-1)^{k / 2}} \int_{S^{2}}(\xi-1)^{k / 2} f\left(\xi-1, \boldsymbol{\omega}^{\prime}\right) d \boldsymbol{\omega}^{\prime} \\
& +\frac{n_{1}}{4 \pi} \sqrt{\frac{\xi+1}{\xi}} \nu(\xi+1) \frac{\xi^{k / 2}}{(\xi+1)^{k / 2}} \int_{S^{2}}(\xi+1)^{k / 2} f\left(\xi+1, \boldsymbol{\omega}^{\prime}\right) d \boldsymbol{\omega}^{\prime}
\end{aligned}
$$


Thus, since $C^{e}$ is conservative, we have

$$
\int_{\mathbb{R}^{3}}\left(\xi^{k / 2} C^{e} f\right)(\xi, \boldsymbol{\omega}) d \boldsymbol{\xi}=\int_{\mathbb{R}^{3}} C^{e}\left(\xi^{k / 2} f\right)(\xi, \boldsymbol{\omega}) d \boldsymbol{\xi}=0,
$$

whereas for the inelastic scattering term we obtain

$$
\begin{aligned}
\int_{\mathbb{R}^{3}}\left(\xi^{k / 2} C_{-}^{i} f\right)(\xi, \boldsymbol{\omega}) d \boldsymbol{\xi} & \\
& =n_{1} \int_{\mathbb{R}^{3}} \nu(\xi) H(\xi-1)\left(\frac{(\xi-1)^{k / 2}}{\xi^{k / 2}}-1\right) \xi^{k / 2} f\left(\xi, \boldsymbol{\omega}^{\prime}\right) d \boldsymbol{\xi} .
\end{aligned}
$$

and similarly,

$$
\int_{\mathbb{R}^{3}}\left(\xi^{k / 2} C_{+}^{i} f\right)(\xi, \boldsymbol{\omega}) d \boldsymbol{\xi}=n_{2} \int_{\mathbb{R}^{3}} \nu(\xi+1) \sqrt{\frac{\xi+1}{\xi}}\left(\frac{(\xi+1)^{k / 2}}{\xi^{k / 2}}-1\right) \xi^{k / 2} f\left(\xi, \boldsymbol{\omega}^{\prime}\right) d \boldsymbol{\xi} .
$$

To see better the meaning of the above formulas, let us take $k=2, \nu=1$ and $f$ independent of $\boldsymbol{\omega}, f(\xi, \boldsymbol{\omega})=f(\xi)$. Then we obtain that

$$
\int_{\mathbb{R}^{3}} \xi^{k / 2}\left(C^{i} f\right)(\xi, \boldsymbol{\omega}) d \boldsymbol{\xi}=n_{2} \int_{0}^{\infty} \sqrt{\xi+1} f(\xi) d \xi-n_{1} \int_{1}^{\infty} \sqrt{\xi} f(\xi) d \xi .
$$

Clearly, if $f$ has the support in the unit ball, then the right-hand side is positive. On the other hand, if the function $f$ has the support outside the ball of radius $r>n_{2}^{2} /\left(n_{1}^{2}-n_{2}^{2}\right)$ (recall that $\left.n_{2}<n_{1}\right)$, then the right-hand side is negative. Therefore $C^{i}$ is not dissipative, let alone conservative, in $X_{k}$.

Due to the translational character of the operator $C^{i}$ it is convenient to introduce the reduced energy $\xi \in[0,1$ [ and to re-define all the functions as functional sequences in the following way: for $n=0,1,2 \ldots$ and $\xi \in[0,1[$

$$
\begin{aligned}
f_{n}(\xi, \boldsymbol{\omega}) & =f(\xi+n, \boldsymbol{\omega}), \\
\lambda_{n}(\xi) & =\lambda(\xi+n), \\
\nu_{n}(\xi) & =\nu(\xi+n), \\
\xi_{n} & =\sqrt{\xi+n} \\
p_{n}(\xi) & =\frac{\xi_{n+1}}{\xi_{n}} \nu_{n+1}(\xi) \\
F_{n}(\xi) & =\frac{1}{4 \pi} \int_{S^{2}} f_{n}(\xi, \boldsymbol{\omega}) d \boldsymbol{\omega} .
\end{aligned}
$$


With this notation the equation (2.6) can be written in the recursive form

$$
\begin{aligned}
\partial_{t} f_{0}= & -\left(\lambda_{0}+n_{2} p_{0}\right) f_{0}+\lambda_{0} F_{0}+n_{1} p_{0} F_{1} \\
\vdots & \vdots \\
\partial_{t} f_{n}= & -\left(\lambda_{n}+n_{2} p_{n}+n_{1} \nu_{n}\right) f_{n}+\lambda_{n} F_{n}+n_{1} p_{n} F_{n+1}+n_{2} \nu_{n} F_{n-1} \\
\vdots & \vdots
\end{aligned}
$$

where $n=1,2, \ldots$ In this notation the $X$ norm of $f$ is finite if and only if

$$
\sum_{j=0}^{\infty} \int_{S^{2}}\left(\int_{0}^{1} \xi_{j}\left|f_{j}(\xi, \boldsymbol{\omega})\right| d \xi\right) d \boldsymbol{\omega}
$$

and $f \in D(C)$ if additionally

$$
\int_{S^{2}} \int_{0}^{1}\left|f_{0}(\xi, \boldsymbol{\omega})\right| d \xi d \boldsymbol{\omega}<\infty
$$

Similarly, $f \in X_{k}$, that is, $f$ has the $k$-th moment finite, if

$$
\begin{aligned}
\|f\|_{k} & =\int_{S^{2}}\left(\int_{0}^{1} \xi_{0}\left|f_{0}(\xi)\right| d \xi+\sum_{j=1}^{\infty} \int_{0}^{1} j^{k / 2}\left|\xi_{j} f_{j}(\xi, \boldsymbol{\omega})\right| d \xi\right) d \boldsymbol{\omega} \\
& <\infty
\end{aligned}
$$

Define $D_{k}(C)$ as the domain of the part of $C$ in $X_{k}$; then $f \in D_{k}(C)$ if and only if

$$
\int_{S^{2}}\left(\int_{0}^{1}\left|f_{0}(\xi)\right| d \xi+\sum_{j=1}^{\infty} j^{k / 2} \int_{0}^{1} \xi_{j}\left|f_{j}(\xi, \boldsymbol{\omega})\right| d \xi\right) d \boldsymbol{\omega}<\infty .
$$

In particular, using the same argument as for $D(C)$, we find that $D_{k}(C)$ is dense in $X_{k}$ for any $k \geq 0$.

We see that the norms in $X_{k}$ consist of two parts: one is the integral over $S^{2} \times[0,1]$, the other is the summation. It will simplify the notation if we introduce for $f=\left(f_{j}\right)_{j \in \mathbb{N}}$

$$
|f|_{k}=\left|f_{0}\right| \xi_{0}+\sum_{j=1}^{\infty} j^{k / 2} \xi_{j}\left|f_{j}\right| .
$$

Thus, the norm in $X_{k}$ is given by

$$
\|f\|_{k}=\int_{S^{2}} \int_{0}^{1}|f|_{k} d \xi d \omega
$$




\section{Generation of semigroup in $X_{k}$}

In this section we shall prove the main generation result.

Theorem 3.1. The operator $C=C^{i}+C^{e}$ with the domain defined by the condition (2.13) generates a positive semigroup in $X_{k}$, for any $k \in \mathbb{N}$.

The proof of this theorem is quite long so that we will split it into a sequence of lemmas. Let us first introduce the notation

$$
\begin{aligned}
\left(K^{e} f\right)(\xi, \boldsymbol{\omega}) & =\frac{1}{4 \pi} \lambda(\xi) \int_{S^{2}} f\left(\xi, \boldsymbol{\omega}^{\prime}\right) d \boldsymbol{\omega}^{\prime} \\
(\mathcal{A} f)(\xi, \boldsymbol{\omega}) & =-\left(n_{1} H(\xi-1) \nu(\xi)+\lambda(\xi)\right) f(\xi, \boldsymbol{\omega}),
\end{aligned}
$$

so that

$$
C f=C_{+}^{i} f+\mathcal{A} f+K_{-}^{i} f+K^{e} f,
$$

where the operators $C_{+}^{i}$ and $K_{-}^{i}$ were introduced in Eqs. (2.4) and (2.5).

Lemma 3.1. The resolvent $R\left(s, C_{+}^{i}+\mathcal{A}\right)$ of the operator $C_{+}^{i}+\mathcal{A}$ with domain $D_{k}\left(C_{+}^{i}+\mathcal{A}\right)=D_{k}(C)$ exists in $X_{k}$ for any $s>0$ and is a positive operator.

Proof. To find the resolvent of $R\left(s, C_{+}^{i}+\mathcal{A}\right)$ we use the representation $(2.9)$ obtaining the system of equations

$$
\begin{aligned}
g_{0}= & \left(s+\lambda_{0}+n_{2} p_{0}\right) f_{0} \\
\vdots & \vdots \\
g_{n}= & \left(s+\lambda_{n}+n_{1} \nu_{n}+n_{2} p_{n}\right) f_{n}-n_{2} \nu_{n} F_{n-1} \\
\vdots & \vdots
\end{aligned}
$$

where $n=1,2, \ldots$.

For $n=0,1, \ldots$, we denote $r_{n}=\lambda_{n}+\left(1-\delta_{n 0}\right) n_{1} \nu_{n}$, where $\delta_{i j}$ is the Kronecker delta. Thus

$$
0<\lambda_{\min } \leq r_{n}(\xi) \leq \lambda_{\max }+n_{1} \nu_{\max }
$$

uniformly in $\xi \in[0,1[$ and $n \geq 0$. 
Let us split all the functions as $g_{k}=g_{k 0}+g_{k 1}$, where $\int_{S^{2}} g_{k 1} d \boldsymbol{\omega}=0$. Thanks to this, upon integration with respect to $d \boldsymbol{\omega}$, the system (3.1) decouples into

$$
\begin{aligned}
g_{00}= & \left(s+r_{0}+n_{2} p_{0}\right) f_{00} \\
\vdots & \vdots \\
g_{n 0}= & \left(s+r_{n}+n_{2} p_{n}\right) f_{n 0}-n_{2} \nu_{n} f_{n-1,0} \\
\vdots & \vdots
\end{aligned}
$$

and

$$
\begin{aligned}
g_{01}= & \left(s+r_{0}+n_{2} p_{0}\right) f_{01} \\
\vdots & \vdots \\
g_{n 1}= & \left(s+r_{n}+n_{2} p_{n}\right) f_{n 1} \\
\vdots & \vdots
\end{aligned}
$$

where $n=1,2, \ldots$. The second system is diagonal, so its solvability renders no difficulties. Let us concentrate then on (3.3). We claim that for $n=$ $0,1 \ldots$ and $s>0$

$$
\begin{aligned}
f_{n 0}= & \frac{1}{\xi_{n}\left(n_{2} p_{n}+r_{n}+s\right)} \\
& \cdot \sum_{i=0}^{n} \xi_{i} g_{i 0}\left(\prod_{l=0}^{n-i-1} \frac{n_{2} p_{n-l-1}}{n_{2} p_{n-l-1}+r_{n-l-1}+s}\right),
\end{aligned}
$$

with the convention that the product is equal to 1 if $n-i-1<0$. In fact, for $n=0$ the formula (3.5) gives

$$
\begin{aligned}
f_{00} & =\frac{1}{\xi_{0}\left(n_{2} p_{0}+r_{0}+s\right)} \sum_{i=0}^{0} \xi_{i} g_{i 0}\left(\prod_{l=0}^{-i-1} \frac{n_{2} p_{n-l-1}}{n_{2} p_{n-l-1}+r_{n-l-1}+s}\right) \\
& =\frac{g_{00}}{n_{2} p_{0}+r_{0}+s}
\end{aligned}
$$

which agrees with the direct solution to the first equation of (3.3). The basic recurrence formula which will enable us to prove (3.5) is

$$
\frac{\xi_{n}}{\xi_{n+1}} p_{n}=\nu_{n+1}, \quad n=0,1 \ldots
$$


Assuming that (3.5) is correct for $n-1$, we find from (3.3) that

$$
\begin{aligned}
f_{n 0}= & \frac{1}{n_{2} p_{n}+r_{n}+s}\left(g_{n 0}+n_{2} \nu_{n} f_{n-1,0}\right) \\
= & \frac{1}{\xi_{n}\left(n_{2} p_{n}+r_{n}+s\right)}\left(\xi_{n} g_{n 0}\right. \\
& \left.+\frac{n_{2} p_{n-1}}{n_{2} p_{n-1}+r_{n-1}+s} \sum_{i=0}^{n-1} \xi_{i} g_{i 0} \prod_{l=0}^{n-i-2} \frac{n_{2} p_{n-l-2}}{n_{2} p_{n-l-2}+r_{n-l-2}+s}\right) \\
= & \frac{1}{\xi_{n}\left(n_{2} p_{n}+r_{n}+s\right)}\left(\xi_{n} g_{n 0}+\sum_{i=0}^{n-1} \xi_{i} g_{i 0} \prod_{l=0}^{n-i-1} \frac{n_{2} p_{n-l-1}}{n_{2} p_{n-l-1}+r_{n-l-1}+s}\right) \\
= & \frac{1}{\xi_{n}\left(n_{2} p_{n}+r_{n}+s\right)} \sum_{i=0}^{n} \xi_{i} g_{i 0} \prod_{l=0}^{n-i-1} \frac{n_{2} p_{n-l-1}}{n_{2} p_{n-l-1}+r_{n-l-1}+s} .
\end{aligned}
$$

Since

$$
p_{i}(\xi)=\frac{\sqrt{\xi+i+1}}{\sqrt{\xi+i}} \nu(\xi+i+1)
$$

we have $\nu_{\min } \leq p_{0} \leq \infty$ and $\nu_{\text {min }} \leq p_{i} \leq \sqrt{2} \nu_{\text {max }}$ uniformly for $\xi \in[0,1[$ and $i \geq 1$. Since the function $\mathbb{R}_{+} \ni r \rightarrow r /(r+s)$ is increasing for any $s>0$, we obtain

$$
\begin{aligned}
\frac{n_{2} p_{0}}{n_{2} p_{0}+s} \leq & 1 \\
\vdots & \vdots \\
\frac{n_{2} p_{i}}{n_{2} p_{i}+r_{i}+s} \leq & \frac{\sqrt{2} n_{2} \nu_{\max }}{\sqrt{2} n_{2} \nu_{\max }+\lambda_{\min }+s} \\
\vdots & \vdots
\end{aligned}
$$

For $s>0$ we denote $\beta=\sqrt{2} n_{2} \nu_{\max } /\left(\sqrt{2} n_{2} \nu_{\max }+\lambda_{\min }+s\right)<1$. Thus

$$
\prod_{l=0}^{n-i-1} \frac{n_{2} p_{n-l-1}}{n_{2} p_{n-l-1}+r_{n-l-1}+s} \leq \beta^{n-i}
$$

for $i=0, \ldots, n-1$, and for $i=n, \prod_{l=0}^{-1} n_{2} p_{n-l-1} /\left(n_{2} p_{n-l-1}+r_{n-l-1}+s\right)=$ 1 by definition. Hence

$$
\begin{aligned}
\xi_{n}\left|f_{n 0}\right| & =\frac{1}{n_{2} p_{n}+r_{n}+s}\left|\sum_{i=0}^{n-1} \xi_{i} g_{i 0} \prod_{l=0}^{n-i-1} \frac{n_{2} p_{n-l-1}}{n_{2} p_{n-l-1}+r_{n-l-1}+s}+\xi_{n} g_{n 0}\right| \\
& \leq \frac{1}{s} \sum_{i=0}^{n-1} \xi_{i 0}\left|g_{i 0}\right| \beta^{n-i}+\xi_{n}\left|g_{n 0}\right|
\end{aligned}
$$


By (2.13) we have to prove that the series

$$
\xi_{0}\left|f_{00}\right|+\sum_{n=1}^{\infty} n^{k / 2} \xi_{n}\left|f_{n 0}\right|
$$

is integrable over $[0, \xi[$. To do this, it is enough to prove the statement for any remainder of the series. Let us fix $N>1$ (which will be determined later) and consider

$$
\begin{aligned}
\sum_{n=N}^{\infty} n^{k / 2} \xi_{n}\left|f_{n 0}\right| & \leq \frac{1}{s} \sum_{n=N}^{\infty}\left(\sum_{i=0}^{n-1} n^{k / 2} \xi_{i}\left|g_{i 0}\right| \beta^{n-i}+n^{k / 2} \xi_{n}\left|g_{n 0}\right|\right) \\
& =\frac{1}{s}\left(\sum_{i=0}^{\infty} \xi_{i}\left|g_{i 0}\right| \beta^{-i}\left(\sum_{n=N+i}^{\infty} n^{k / 2} \beta^{n}\right)+\sum_{i=N}^{\infty} i^{k / 2} \xi_{i}\left|g_{i 0}\right|\right)
\end{aligned}
$$

where the change of the order of summation is justified by the positivity of the terms.

Consider now the function $r \rightarrow r^{k / 2} \beta^{r}$. This function is monotonically decreasing for $r>r_{0}=-k(2 \ln \beta)^{-1}$. Taking $N \geq \max \left\{r_{0}, 1-(\ln \beta)^{-1}\right\}$ we have the estimate

$$
\sum_{n=N+i}^{\infty} n^{k / 2} \beta^{n} \leq \int_{N+i-1}^{\infty} r^{k / 2} \beta^{r} d r
$$

To shorten notation, let $\gamma=\beta^{-1}$. Then

$$
\int_{N+i-1}^{\infty} r^{k / 2} \beta^{r} d r=\int_{N+i-1}^{\infty} r^{k / 2} \gamma^{-r} d r=(\ln \gamma)^{-(1+k / 2)} \int_{\ln \gamma(N+i-1)}^{\infty} z^{k / 2} e^{-z} d z
$$

and the last integral is the well known incomplete $\Gamma$ function. From [1], formula 6.5.32, we infer that for some $M_{k}$ which is independent of the lower limit of integration $z \geq 1$ (but dependent on $k$ ) we have

$$
\int_{z}^{\infty} z^{k / 2} e^{-z} d z \leq M_{k} z^{k / 2} e^{-z}
$$

Because of the definition of $N$, for any $\gamma$ and $i \geq 0$ the lower limit of integration satisfies $(N+i-1) \ln \gamma \geq 1$, thus we can use the estimate (3.10) 
to obtain

$$
\begin{aligned}
\sum_{n=N+i}^{\infty} n^{k / 2} \beta^{n} & \leq(\ln \gamma)^{-(1+k / 2)} \int_{\ln \gamma(N+i-1)}^{\infty} z^{k / 2} e^{-z} d z \\
& \leq M_{k}(\ln \gamma)^{-1}(N+i-1)^{k / 2} \beta^{N+i-1} \\
& \leq M_{k}(\ln \gamma)^{-1}(N+i-1)^{k / 2} \beta^{i}
\end{aligned}
$$

where we used the fact that $N-1>0$ and $\beta<1$, so that $\beta^{N-1}<1$. Since $i^{-k / 2}(N+i-1)^{k / 2} \leq(N+1)^{k / 2}$ for $i \geq 1$ and also $(N-1)^{k / 2} \leq(N+1)^{k / 2}$, we obtain

$$
\begin{aligned}
\sum_{n=N}^{\infty} n^{k / 2} \xi_{n}\left|f_{n 0}\right| \leq & \frac{M_{k}}{s \ln \gamma}\left(\xi_{0}\left|g_{00}\right|(N-1)^{k / 2}+\sum_{i=1}^{\infty} \xi_{i}(N+i-1)^{k / 2}\left|g_{i 0}\right|\right. \\
& \left.+\sum_{i=N}^{\infty} i^{k / 2} \xi_{i}\left|g_{i 0}\right|\right) \\
\leq & \frac{M_{k}(N+1)^{k / 2}}{s \ln \gamma}\left(\xi_{0}\left|g_{00}\right|+\sum_{i=1}^{\infty} \xi_{i} i^{k / 2}\left|g_{i 0}\right|+\sum_{i=1}^{\infty} i^{k / 2} \xi_{i}\left|g_{i 0}\right|\right) \\
= & \frac{2 M_{k}(N+1)^{k / 2}}{s \ln \gamma}\left|g_{0}\right| k,
\end{aligned}
$$

where $g_{0}=\left\{g_{i 0}\right\}_{i \in \mathbb{N}}$. For the initial part of the series (3.9) we have

$$
\begin{aligned}
\sum_{n=1}^{N-1} n^{k / 2} \xi_{n}\left|f_{n 0}\right| & \leq \frac{1}{s} \sum_{n=1}^{N-1}\left(\sum_{i=0}^{n-1} n^{k / 2} \xi_{i}\left|g_{i 0}\right| \beta^{n-i}+n^{k / 2} \xi_{n}\left|g_{n}\right|\right) \\
& \leq \frac{1}{s}\left|g_{0}\right|_{k}\left(\sum_{n=1}^{N-1} n^{k / 2}(n-1)\right) \\
& \leq \frac{M_{k}^{\prime} N^{2+k / 2}}{s}\left|g_{0}\right|_{k},
\end{aligned}
$$

where $M_{k}^{\prime}$ is independent of $\beta$ and $N$, and where we used the fact that $n-i>0$ for $0 \leq i \leq n-1$, so that $\beta^{n-i}<1$ in the above series. The final inequality was obtained by integration. Moreover

$$
f_{00}=\frac{g_{00}}{n_{2} p_{0}+s}=\frac{\xi_{0} g_{00}}{s \xi_{0}+\xi_{1} n_{2} \nu_{1}}
$$

which, on one hand, shows that $f_{0} \in D_{k}(C)$ and on the other, gives the estimate

$$
\left|\xi_{0} f_{00}\right| \leq \frac{1}{s} \xi_{0}\left|g_{00}\right|
$$


Combining all the estimates we see that for any $s>0$ there exists a constant

$$
M_{k, s}=2 M_{k}(\ln \gamma)^{-1}(N+1)^{k / 2}+M_{k}^{\prime} N^{2+k / 2}+1
$$

(depending on $s$ and $k$ ) such that

$$
\left\|f_{0}\right\|_{k} \leq \frac{M_{k, s}}{s}\left\|g_{0}\right\|_{k}
$$

and $f_{0} \in D_{k}(C)$ because of (3.12). Since (3.4) is diagonal, we immediately obtain for $i \geq 0$

$$
\left|f_{i 1}\right|=\left|\frac{g_{i 1}}{s+n_{2} p_{i}}\right| \leq \frac{\left|g_{i 1}\right|}{s}
$$

thus

$$
\left|f_{1}\right|_{k} \leq \frac{1}{s}\left|g_{1}\right|_{k}
$$

and due to

$$
\left|f_{01}\right|=\left|\frac{g_{01}}{s+n_{2} p_{0}}\right|=\left|\frac{\xi_{0} g_{01}}{\xi_{0} s+n_{2} \nu_{1} \xi_{1}}\right| \leq\left|\frac{\xi_{0} g_{01}}{n_{2} \nu_{1} \xi_{1}}\right|
$$

we see that $f_{1} \in D_{k}(C)$. To obtain the estimate valid for $f=f_{0}+f_{1}$ let us recall that we have decomposed $X_{k}$ into the direct sum $X_{k}=X_{k 0} \oplus X_{k 1}$, where any $g_{0} \in X_{k 1}$ satisfies $\int_{S^{2}} g_{0} d \boldsymbol{\omega}=0$. Let $P_{0}$ and $P_{1}=I-P_{0}$ be the complementary projections onto $X_{k 0}$ and $X_{k 1}$, respectively. We may take $P_{0} g=(4 \pi)^{-1} \int_{S^{2}} g d \boldsymbol{\omega}$ and $P_{1} g=g-(4 \pi)^{-1} \int_{S^{2}} g d \boldsymbol{\omega}$. It is well known that the norm of $g$ in $X_{k}$ is equivalent to $\left\|P_{0} g\right\|_{k}+\left\|P_{1} g\right\|_{k}$. In our case we have for instance

$$
\|g\|_{k} \leq\left\|P_{0} g\right\|_{k}+\left\|P_{1} g\right\|_{k}
$$

and

$$
\left\|P_{0} g\right\|_{k} \leq\|g\|_{k}, \quad\left\|P_{1} g\right\|_{k}=\left\|\left(I-P_{0}\right) g\right\|_{k} \leq 2\|g\|_{k} .
$$

Combining (3.14) and (3.15) with the above we obtain

$$
\begin{aligned}
\|f\|_{k} & \leq\left\|f_{0}\right\|_{k}+\left\|f_{1}\right\|_{k} \leq \frac{M_{k, s}}{s}\left\|g_{0}\right\|_{k}+\frac{1}{s}\left\|g_{1}\right\|_{k} \\
& \leq \frac{M_{k, s}+2}{s}\|g\|_{k} .
\end{aligned}
$$

This shows that the resolvent set of $C_{+}^{i}+\mathcal{A}$ satisfies $\rho\left(C_{+}^{i}+\mathcal{A}\right) \supset\{s \in \mathbb{R} ; s>$ $0\}$. It remains to prove that $R\left(s, C_{+}^{i}+\mathcal{A}\right)$ is a positive operator for $s>0$. Let us take $g \geq 0$. Unfortunately, while $g_{0}=P_{0} g=(4 \pi)^{-1} \int_{S^{2}} g d \boldsymbol{\omega} \geq 0$, the complement $g_{1}$ is not necessarily nonnegative. However, as in (3.8) we have

$$
f_{n 0}=\frac{1}{\xi_{n}\left(n_{2} p_{n}+r_{n}+s\right)}\left(\sum_{i=0}^{n-1} \xi_{i} g_{i 0} \prod_{l=0}^{n-i-1} \frac{n_{2} p_{n-l-1}}{n_{2} p_{n-l-1}+r_{n-l-1}+s}+\xi_{n} g_{n 0}\right)
$$


and from (3.4)

$$
f_{n 1}=\frac{1}{n_{2} p_{n}+r_{n}+s} g_{n 1}=\frac{1}{n_{2} p_{n}+r_{n}+s}\left(g_{n}-g_{n 0}\right) .
$$

Adding these two together we have

$$
\begin{aligned}
f_{n}= & \frac{1}{\xi_{n}\left(n_{2} p_{n}+r_{n}+s\right)}\left(\frac{1}{s} \sum_{i=0}^{n-1} \xi_{i} g_{i 0} \prod_{l=0}^{n-i-1} \frac{n_{2} p_{n-l-1}}{n_{2} p_{n-l-1}+r_{n-l-1}+s}\right) \\
& +\frac{1}{n_{2} p_{n}+r_{n}+s} g_{n}
\end{aligned}
$$

and since $g_{n}, g_{n 0} \geq 0$, we obtain that $f_{n} \geq 0$ and $C_{+}^{i}+\mathcal{A}$ is resolvent positive for $s>0$.

Proof of Theorem 3.1. We write the operator $C_{+}^{i}+\mathcal{A}$ as the sum of the operator $\mathcal{M}$ of multiplication by the function

$$
-\left(n_{2} \sqrt{\frac{\xi+1}{\xi}} \nu(\xi+1)+n_{1} H(\xi-1) \nu(\xi)+\lambda(\xi)\right)
$$

and the operator $K_{+}^{i}$. It is clear that the operator $\mathcal{M}$ defined on $D_{k}(C)$ generates a positive semigroup. Since $K_{+}^{i}$ is a positive bounded linear operator from $D_{k}(C)$ into $X_{k}$, and from Lemma 3.1 we see that the resolvent of $C_{+}^{i}+\mathcal{A}=\mathcal{M}+K_{+}^{i}$ exists and is positive, we can apply Desch's perturbation theorem (see e.g. [22], Theorem 8.1) showing that $C_{+}^{i}+\mathcal{A}$ generates a positive semigroup. To complete the proof we note that $K_{-}^{i}+K^{e}$ is a positive and bounded operator, hence the operator $C=C_{+}^{i}+\mathcal{A}+K_{-}^{i}+K^{e}$, defined on the domain $D_{k}(C)$, generates a positive strongly continuous semigroup.

Remark 3.1. We should point out that the presented method is by no means a unique way of proving the existence of the moments in our particular problem. In such a simplified case one can prove that the operator $K_{+}^{i}$ is bounded in $D(C)$ (see [5], Eq. (4.4)) which allows to use another Desch's result ([15], p. 149) to claim that $A+K_{+}^{i}$ generates a semigroup. We have chosen the above, longer but more direct, method which turns to have a wider range of applicability, [6]. 


\section{An application to asymptotic analysis of kinetic equation with dominant elastic scattering}

Let us consider the Cauchy problem for the kinetic equation

$$
\begin{aligned}
\partial_{t} f_{\varepsilon} & =S f_{\varepsilon}+C^{i} f_{\varepsilon}+\frac{1}{\varepsilon} C^{e} f_{\varepsilon} \\
f_{\varepsilon}(0) & =\stackrel{\circ}{f},
\end{aligned}
$$

where $S$ is the streaming operator defined by $S f=\boldsymbol{v} \cdot \partial_{\boldsymbol{x}} f$ and $C^{i}$ and $C^{e}$ are inelastic and elastic scattering operators defined by (2.2) and (2.1), respectively. The presence of the large factor $1 / \varepsilon$ accounts for the fact that we analyze the case when the elastic collisions are dominant or, in other words, that the mean free path between elastic collisions is very small. A detailed explanation of the above scaling can be found in $[14,7]$. Our aim here is to find the limit equation as $\varepsilon \rightarrow 0$ and to prove that the solutions to the limit equation are limits of the solutions to (4.1) in $L_{1}$ norm.

In this section the problem becomes dependent on $\boldsymbol{x} \in \mathbb{R}^{3}$. Accordingly, we introduce the notation

$$
\mathcal{X}=L_{1}\left(\mathbb{R}_{x}^{3}, X\right)=L_{1}\left(\mathbb{R}_{x, v}^{6}\right)
$$

and

$$
\mathcal{X}_{k}=L_{1}\left(\mathbb{R}_{x}^{3}, X_{k}\right)=L_{1}\left(\mathbb{R}_{x, v}^{6},\left(1+v^{k}\right) d \boldsymbol{v} d \boldsymbol{x}\right)
$$

\subsection{Formal asymptotic results.}

In this subsection we shall carry out the formal asymptotic analysis of the equation (4.1) so that it becomes clear what assumptions are needed for the availability of the rigorous asymptotic procedure. The properties of $C^{e}$

are important even at this formal stage so that we recall the theorem from [5] which provides all the necessary information.

Theorem 4.1. Let all the assumptions of Section 2 are satisfied. Then the operator $C^{e}$ is a bounded operator in $\mathcal{X}$ with the following properties

(i) For any $f \in \mathcal{X}$ and any non-decreasing function $\kappa$ we have

$$
\int_{\mathbb{R}^{6}}\left(\kappa(f) C^{e} f\right)(\boldsymbol{x}, \boldsymbol{v}) d \boldsymbol{x} d \boldsymbol{v} \leq 0 .
$$


(ii) The null-space $N\left(C^{e}\right)$, the range $R\left(C^{e}\right)$ and the spectral projection $\mathbb{P} f$ onto $N\left(C^{e}\right)$ are given by

$$
\begin{aligned}
N\left(C^{e}\right) & =\{f \in \mathcal{X} ; f \text { is independent on } \boldsymbol{\omega}\} . \\
R\left(C^{e}\right) & =W=\left\{f \in \mathcal{X} ; \int_{S^{2}} f(\boldsymbol{x}, v \boldsymbol{\omega}) d \boldsymbol{\omega}=0 \text { a.e. }\right\} . \\
(\mathbb{P} f)(\boldsymbol{x}, v) & =\frac{1}{4 \pi} \int_{S^{2}} f(\boldsymbol{x}, v \boldsymbol{\omega}) d \boldsymbol{\omega} .
\end{aligned}
$$

(iii) For $f \in W$ we have

$$
\int_{\mathbb{R}^{6}}\left(\operatorname{sign}(f) C^{e} f\right)(\boldsymbol{x}, \boldsymbol{v}) d \boldsymbol{x} d \boldsymbol{v} \leq-4 \pi \lambda_{\min }\|f\|_{\mathcal{X}} .
$$

Remark 4.1. Theorem 4.1 was proved under more general assumptions than that of Section 2. In the present context all the statements can be verified by direct computations as was done in [14]. In particular, we can derive the explicit expression for the inverse of $\mathbb{Q} C^{e} \mathbb{Q}$, where $\mathbb{Q}=I-\mathbb{P}$. In fact, from (2.1) we obtain immediately $C^{e} \mathbb{Q} f=-\lambda f$. If $f \in W$, then $-\lambda f \in W$, thus $\mathbb{Q} C^{e} \mathbb{Q} f=-\lambda f$. Consequently,

$$
\left(\mathbb{Q} C^{e} \mathbb{Q}\right)^{-1} f=-\lambda^{-1} f .
$$

The asymptotic procedure we are going to use is fairly well known, e.g. $[21,16]$. We operate on both sides of $(4.1)$ with the projections $\mathbb{P}$ and $\mathbb{Q}$ and, denoting $f_{\varepsilon}=\mathbb{P} f_{\varepsilon}+\mathbb{Q} f_{\varepsilon}=v_{\varepsilon}+w_{\varepsilon}$, we obtain the following system

$$
\begin{aligned}
\partial_{t} v_{\varepsilon} & =\mathbb{P} S \mathbb{P} v_{\varepsilon}+\mathbb{P} S \mathbb{Q} w_{\varepsilon}+\mathbb{P} C^{i} \mathbb{P} v_{\varepsilon}+\mathbb{P} C^{i} \mathbb{Q} w_{\varepsilon} \\
\partial_{t} w_{\varepsilon} & =\mathbb{Q} S \mathbb{P} v_{\varepsilon}+\mathbb{Q} S \mathbb{Q} w_{\varepsilon}+\mathbb{Q} C^{i} \mathbb{P} v_{\varepsilon}+\mathbb{Q} C^{i} \mathbb{Q} w_{\varepsilon}+\frac{1}{\varepsilon} \mathbb{Q} C^{e} \mathbb{Q} w_{\varepsilon}
\end{aligned}
$$

where we have already used the fact that $\mathbb{P} C^{e} \equiv 0$. The system has to be supplemented by the initial values

$$
\begin{aligned}
& v_{\varepsilon}(0)=\mathbb{P} \stackrel{\circ}{f}=\stackrel{\circ}{v} \\
& w_{\varepsilon}(0)=\mathbb{Q} \stackrel{\circ}{f}=\stackrel{\circ}{w} .
\end{aligned}
$$

Isotropic form of the scattering operators allows us to simplify system (4.8) even further. To do this we note that the operator $C^{i}$ reduces $N\left(C^{e}\right)$ and $W=N\left(C^{e}\right)^{\perp}$, thus $\mathbb{P} C^{i} \mathbb{Q}=\mathbb{Q} C^{i} \mathbb{P}=0$. Moreover, $S \mathbb{P} v_{\varepsilon}$ is linear in $\boldsymbol{\omega}$, therefore $\mathbb{P} S \mathbb{P} v_{\varepsilon}=0$. Taking these into account we transform (4.8) into the 
following form

$$
\begin{aligned}
\partial_{t} v_{\varepsilon} & =\mathbb{P} S \mathbb{Q} w_{\varepsilon}+\mathbb{P} C^{i} \mathbb{P} v_{\varepsilon} \\
\partial_{t} w_{\varepsilon} & =\mathbb{Q} S \mathbb{P} v_{\varepsilon}+\mathbb{Q} S \mathbb{Q} w_{\varepsilon}+\mathbb{Q} C^{i} \mathbb{Q} w_{\varepsilon}+\frac{1}{\varepsilon} \mathbb{Q} C^{e} \mathbb{Q} w_{\varepsilon}
\end{aligned}
$$

The asymptotic solution is sought in the form

$$
\begin{aligned}
f(t, t / \varepsilon)= & \bar{f}(t)+\tilde{f}(t / \varepsilon)=\rho(t)+\bar{w}_{0}(t)+\varepsilon \bar{w}_{1}(t)+\ldots \\
& +\tilde{\rho}_{0}(t / \varepsilon)+\varepsilon \tilde{\rho}_{1}(t / \varepsilon)+\ldots+\tilde{w}_{0}(t / \varepsilon) \\
& +\varepsilon \tilde{w}_{1}(t / \varepsilon)+\ldots
\end{aligned}
$$

where

$$
\rho, \tilde{\rho}_{0}, \tilde{\rho}_{1} \ldots \in N\left(C^{e}\right),
$$

and are called the hydrodynamic part of the expansion, whereas

$$
\bar{w}_{0}, \bar{w}_{1}, \ldots, \tilde{w}_{0}, \tilde{w}_{1}, \ldots \in W
$$

and are called the kinetic part of the expansion. Moreover, the terms depending on $t$ are referred to as the bulk part of the asymptotic expansion and the terms depending on $\tau=t / \varepsilon$ are known as the initial layer; they are to be determined independently of each other. Note that in accordance with the Chapman-Enskog (compressed) procedure ([21]) the hydrodynamic term of the bulk part of the expansion, $\rho$, is not expanded. The number of the terms in each expansion is determined in some sense a posteriori after having written the equations for the error. It follows that inserting the expansion (4.10) into (4.9) and equating the coefficients at equal powers of $\varepsilon$ we obtain the following equations:

$$
\begin{aligned}
\partial_{t} \rho & =\mathbb{P} C^{i} \mathbb{P} \rho, \\
\mathbb{Q} C^{e} \mathbb{Q} \bar{w}_{0} & =0, \\
\mathbb{Q} C^{e} \mathbb{Q} \bar{w}_{1}+\mathbb{Q} S \mathbb{P} \rho & =0, \\
\partial_{\tau} \tilde{\rho}_{0} & =0, \\
\partial_{\tau} \tilde{w}_{0} & =\mathbb{Q} C^{e} \mathbb{Q} \tilde{w}_{0},
\end{aligned}
$$

which, as we shall see, define enough terms of the asymptotic expansion to obtain, at least formally, the convergence of the difference $f_{\varepsilon}-f(t, t / \varepsilon)$ to zero as $\varepsilon \rightarrow 0$.

In fact, let us assume for a time being that all the equations above can be solved and that the solutions are sufficiently regular to make the manipulations to follow available. It can be proved that on this level of approximation the correct initial values for (4.11) and (4.13) are

$$
\rho(0)=\stackrel{\circ}{v}, \quad \tilde{w}_{0}(0)=\stackrel{\circ}{w} .
$$


Note that the equation for $\bar{w}_{1}$ is algebraic and does not require any side condition and the solution to (4.12) is determined by the stipulated decay to zero as $\tau \rightarrow \infty$.

Thus we have $\bar{w}_{0}=\tilde{\rho}_{0}=0$ and

$$
\begin{aligned}
& \bar{w}_{1}=-\left(\mathbb{Q} C^{e} \mathbb{Q}\right)^{-1} \mathbb{Q} S \mathbb{P} \rho \\
& \tilde{w}_{0}=e^{\tau \mathbb{Q} C^{e} \mathbb{Q}} \stackrel{\circ}{w} .
\end{aligned}
$$

Hence, we take the pair $\left(\rho, \tilde{w}_{0}+\varepsilon \bar{w}_{1}\right)$ as the approximation of $f_{\varepsilon}=$ $\left(v_{\varepsilon}, w_{\varepsilon}\right)$; the error of this approximation is given by

$$
\begin{aligned}
& y_{\varepsilon}=v_{\varepsilon}-\rho \\
& z_{\varepsilon}=w_{\varepsilon}-\tilde{w}_{0}-\varepsilon \bar{w}_{1} .
\end{aligned}
$$

Assuming that the solution and the terms of the asymptotic expansion are regular enough (we require that they belong to the domains of all the operators involved in (4.9)) and taking into account equations (4.11)-(4.13) we obtain the following system of equations for the error

$$
\begin{gathered}
\partial_{t} y_{\varepsilon}-\mathbb{P} S \mathbb{Q} z_{\varepsilon}-\mathbb{P} C^{i} \mathbb{P} y_{\varepsilon}=\varepsilon \mathbb{P} S \mathbb{Q} \bar{w}_{1}+\mathbb{P} S \mathbb{Q} \tilde{w}_{0} \\
\partial_{t} z_{\varepsilon}-\mathbb{Q} S \mathbb{P} y_{\varepsilon}-\mathbb{Q} S \mathbb{Q} z_{\varepsilon}-\mathbb{Q} C^{i} \mathbb{Q} z_{\varepsilon}-\frac{1}{\varepsilon} \mathbb{Q} C^{e} \mathbb{Q} z=\varepsilon \mathbb{Q} S \mathbb{Q} \bar{w}_{1}+\mathbb{Q} S \mathbb{Q} \tilde{w}_{0} \\
+\varepsilon \mathbb{Q} C^{i} \mathbb{Q} \bar{w}_{1}+\mathbb{Q} C^{i} \mathbb{Q} \tilde{w}_{0}-\varepsilon \partial_{t} \bar{w}_{1},
\end{gathered}
$$

with the initial conditions

$$
\begin{aligned}
& y_{\varepsilon}(0)=0 \\
& z_{\varepsilon}(0)=-\varepsilon \bar{w}_{1}(0)=\varepsilon\left(\mathbb{Q} C^{e} \mathbb{Q}\right)^{-1} \mathbb{Q} S \mathbb{P} \stackrel{\circ}{v} .
\end{aligned}
$$

The next step can be summarized as follows: due to the assumed regularity of all the terms, the error $\left(y_{\varepsilon}, z_{\varepsilon}\right)$ is a classical solution of (4.14). Defining

$$
e_{\varepsilon}=y_{\varepsilon}+z_{\varepsilon}
$$

and $K_{\varepsilon}=S+C^{i}+\frac{1}{\varepsilon} C^{e}$, we see that $e_{\varepsilon}$ is a classical solution to

$$
\begin{aligned}
\partial_{t} e_{\varepsilon}-K_{\varepsilon} e_{\varepsilon} & =\varepsilon\left(S \mathbb{Q} \bar{w}_{1}+\mathbb{Q} C^{i} \mathbb{Q} \bar{w}_{1}-\partial_{t} \bar{w}_{1}\right)+S \mathbb{Q} \tilde{w}_{0}+\mathbb{Q} C^{i} \mathbb{Q} \tilde{w}_{0} \\
e_{\varepsilon}(0) & =\varepsilon\left(\mathbb{Q} C^{e} \mathbb{Q}\right)^{-1} \mathbb{Q} S \mathbb{P} \stackrel{\circ}{v}
\end{aligned}
$$


The semigroup solving this equation is contractive in $\mathcal{X}$, [3], thus using the Duhamel formula we obtain the estimate

$$
\begin{aligned}
\left\|e_{\varepsilon}(t)\right\| \leq & \varepsilon\left\|\left(\mathbb{Q} C^{e} \mathbb{Q}\right)^{-1} \mathbb{Q} S \mathbb{P} \stackrel{\circ}{v}\right\| \\
& +\varepsilon \int_{0}^{t}\left\|S \mathbb{Q} \bar{w}_{1}(s)+\mathbb{Q} C^{i} \mathbb{Q} \bar{w}_{1}(s)-\partial_{s} \bar{w}_{1}(s)\right\| d s \\
& +\int_{0}^{t}\left\|S \mathbb{Q} \tilde{w}_{0}(s / \varepsilon)+\mathbb{Q} C^{i} \mathbb{Q} \tilde{w}_{0}(s / \varepsilon)\right\| d s .
\end{aligned}
$$

From the above inequality we see that if all the expressions in the first two terms exist and are locally bounded in $t$ on $[0, \infty[$, then the contribution of this integral is of order of $\varepsilon$. As far as the second integral is concerned, the initial layer is assumed to be exponentially decaying, that is, to be of order of $e^{-\omega t / \varepsilon}$ for some $\omega>0$. If this property is preserved after having operated on $\tilde{w}_{0}$ with the operators $S \mathbb{Q}$ and $\mathbb{Q} C^{i} \mathbb{Q}$, then upon integration we obtain that also the contribution of this term is of order of $\varepsilon$, thus $\left\|e_{\varepsilon}\right\|=O(\varepsilon)$ and the convergence is proved.

Hence, we see that to complete our considerations we have to prove that all the terms of the asymptotic expansion and the solution to (4.1) are sufficiently regular for all the discussed steps to be permissible. Checking this is the subject of the next subsection.

\subsection{Regularity of the terms of the asymptotic expansion.}

The fact that the operator $K_{\varepsilon}=S+C^{i}+(1 / \varepsilon) C^{e}$ generates a semigroup of contractions has been proved in [3]. Precisely speaking, [3] contains a proof of generation theorem for $S+C^{i}$, but since $C^{e}$ is a bounded operator, the result extends to the present case by the Bounded Perturbation Theorem. Moreover, since $C^{e}$ is a dissipative operator (in fact, it is conservative), the factor $1 / \varepsilon$ does not affect the contractivity of the full semigroup. Under the Maxwell molecule assumption the domain of the generator $D\left(K_{\varepsilon}\right)$ is equal to $D(S) \cap D\left(C^{i}\right)$, [3], thus, if $\stackrel{\circ}{u \in D}\left(K_{\varepsilon}\right)$, then we can write

$$
\partial_{t} f_{\varepsilon}=K_{\varepsilon} f_{\varepsilon}=S f_{\varepsilon}+C^{i} f_{\varepsilon}+\frac{1}{\varepsilon} C^{e} f_{\varepsilon}
$$

We prove the following lemma.

Lemma 4.1. If $\stackrel{\circ}{f} \in D(S) \cap D\left(C^{i}\right)$, then $\mathbb{P} f_{\varepsilon}, \mathbb{Q} f_{\varepsilon} \in D(S) \cap D\left(C^{i}\right)$ and therefore (4.1) and (4.8) are equivalent. 
Proof. Firstly, we prove that $\mathbb{P} u \in D(S)$. Let us recall that

$$
D(S)=\left\{f \in \mathcal{X}, \boldsymbol{v} \cdot \partial_{\boldsymbol{x}} f \in \mathcal{X}\right\},
$$

where the gradient of $f$ is taken in the distributional sense. Clearly, if $f \in \mathcal{X}$, then $\mathbb{P} f \in \mathcal{X}$. Let $\phi$ be any test function. Then

$$
\left\langle\boldsymbol{v} \cdot \partial_{\boldsymbol{x}} \mathbb{P} f, \phi\right\rangle=-\int_{\mathbb{R}_{x, v}^{6}}\left(\int_{S^{2}} f(\boldsymbol{x}, v \boldsymbol{\omega}) d \boldsymbol{\omega}\right) \boldsymbol{v} \cdot \partial_{\boldsymbol{x}} \phi d \boldsymbol{v} d \boldsymbol{x} .
$$

Since $\left(\boldsymbol{\omega}^{\prime}, \boldsymbol{\omega}, v, \boldsymbol{x}\right) \rightarrow f(\boldsymbol{x}, v \boldsymbol{\omega})\left(v \boldsymbol{\omega}^{\prime} \cdot \partial_{\boldsymbol{x}} \phi\left(\boldsymbol{x}, v \boldsymbol{\omega}^{\prime}\right)\right)$ is integrable on $S^{2} \times S^{2} \times$ $\mathbb{R}_{x, v}^{6}$ we can change the order of integration and using the integrability of $\boldsymbol{v} \cdot \partial_{\boldsymbol{x}} f$ over $\mathbb{R}_{x, v}^{6}$, we rewrite the distributional derivative as

$$
\left\langle\boldsymbol{v} \cdot \partial_{\boldsymbol{x}} \mathbb{P} f, \phi\right\rangle=\int_{\mathbb{R}_{x, v}^{6}}\left(\int_{S^{2}} \boldsymbol{v} \cdot \partial_{\boldsymbol{x}} f d \boldsymbol{\omega}\right) \phi d \boldsymbol{x} d \boldsymbol{v},
$$

hence, for any $f \in D(S)$ we have $S \mathbb{P} f=\mathbb{P} S f$ and therefore $\mathbb{P} f \in D(S)$. Since $\mathbb{Q} f=f-\mathbb{P} f$, we have immediately that also $\mathbb{Q} f \in D(S)$.

The fact that $\mathbb{P} f \in D(C)$ is straightforward, as $\mathbb{P}$ commutes with $C^{i}$.

The question of the sufficient regularity of the bulk part of expansion is addressed in the next lemma. Let us first introduce the notation

$$
\mathcal{X}_{l k m}=W_{1}^{l}\left(\mathbb{R}_{x}^{3}, D_{k}\left(\left(C^{i}\right)^{m}\right)\right)
$$

and

$$
\mathcal{D}_{k}\left(\left(C^{i}\right)^{m}\right)=\mathcal{X}_{0 k m}=L_{1}\left(\mathbb{R}_{x}^{3}, D_{k}\left(\left(C^{i}\right)^{m}\right)\right) .
$$

Clearly, if $m=0$ we have

$$
\mathcal{X}_{l k 0}=W_{1}^{l}\left(\mathbb{R}_{x}^{3}, X_{k}\right)
$$

For $m=1$ we see that $f \in \mathcal{X}_{l k 1}$ if $\partial_{x}^{\beta} f \in D_{k}\left(C^{i}\right)$ for $|\beta| \leq l$ and the norm $\boldsymbol{x} \rightarrow\left\|\partial_{x}^{\beta} f(\boldsymbol{x}, \cdot)\right\|_{D_{k}\left(C^{i}\right)}$ is integrable over $\mathbb{R}_{x}^{3}$ or, in other words, it is sufficient that all the derivatives be integrable over $\mathbb{R}_{x, v}^{6}$ with the appropriate moment weight function.

Before we formulate and prove the lemma on the regularity of the bulk solution we note some important observations related to the introduced space. They either belong to the mathematical folklore or are easy to prove.

First of all, it can be proved that $f \in W_{1}^{1}\left(\mathbb{R}_{x}^{n}, L_{1}\left(\mathbb{R}_{v}^{n}, w(\boldsymbol{v}) d \boldsymbol{v}\right)\right)$, where $w$ is a weight function bounded on bounded subsets of $\mathbb{R}_{v}^{3}$, if and only if the difference quotients $\Delta_{j, h} f=h^{-1}\left(f\left(x_{1}, \ldots, x_{j}+h, \ldots, \boldsymbol{v}\right)-f\left(x_{1}, \ldots, x_{n}, \boldsymbol{v}\right)\right)$ converge to $\partial_{x_{j}} f$ in $L_{1}\left(\mathbb{R}_{x, v}^{6}, w(\boldsymbol{v}) d \boldsymbol{v} d \boldsymbol{x}\right)$ as $h \rightarrow 0$. The extension to higher derivatives can be done by induction. 
By direct calculations as in Lemma 4.1 we can prove that if the distributional derivative $\partial_{x_{j}} f \in \mathcal{D}_{k}\left(C^{i}\right)$, then

$$
\partial_{x_{j}} C^{i} f=C^{i} \partial_{x_{j}} f
$$

Consequently, if $\partial_{x_{j}} f \in \mathcal{D}_{k}\left(C^{i}\right)$ for $j=1, \ldots, n$, then $C^{i} f \in W_{1}^{1}\left(\mathbb{R}_{x}^{n}\right.$, $\left.L_{1}\left(\mathbb{R}_{v}^{n}, w(\boldsymbol{v}) d \boldsymbol{v}\right)\right)$. Moreover, in such a case $C^{i} \Delta_{j, h} f \rightarrow C^{i} \partial_{x_{j}} f$ or, in other words

$$
\lim _{h \rightarrow 0^{+}} \Delta_{j} f=\partial_{x_{j}} f, \quad \text { in } \mathcal{D}_{k}\left(C^{i}\right) .
$$

Similar conclusions can be drawn for higher derivatives.

From the above it follows that if $(T(t))_{t \geq 0}$ is the semigroup generated by $C^{i}$ and $\partial_{x_{j}} f \in \mathcal{D}\left(C^{i}\right)$, then $\partial_{x_{j}} T(t) f=T(t) \partial_{x_{j}} f, t \geq 0$. This follows by the following argument. Let $\stackrel{\circ}{f}, \partial_{x_{j}} f \in \mathcal{D}_{k}\left(C^{i}\right)$ and $f$ and $F$ be the respective classical solutions to (2.6). Then $F_{j, h}=\Delta_{j, h} f$ is the classical solution to (2.6) corresponding to the initial value $\Delta_{j, h} \stackrel{\circ}{f}$. Since $\Delta_{j, h} \stackrel{\circ}{f} \rightarrow \partial_{x_{j}} \stackrel{\circ}{f}$, we obtain

$$
\partial_{x_{j}} T(t) \stackrel{\circ}{f}=\lim _{h \rightarrow 0} F_{j, h}(t)=F(t)=T(t) \partial_{x_{j}} \stackrel{\circ}{f} .
$$

Now we are ready to prove the following lemma.

Lemma 4.2. Let $\stackrel{\circ}{v}=\mathbb{P} \stackrel{\circ}{f} \in \mathcal{X}_{221}=W_{1}^{2}\left(\mathbb{R}_{x}^{3}, D_{2}\left(C^{i}\right)\right)$. Then for each interval $[0, T], 0<T<+\infty$, there exists a constant $M_{T}$ such that

$$
\begin{aligned}
& \left\|\left(\mathbb{Q} C^{e} \mathbb{Q}\right)^{-1} \mathbb{Q} S \mathbb{P} \stackrel{\circ}{v}\right\|_{\mathcal{X}} \\
& \quad+\max _{t \in[0, T]}\left\{\left\|S \mathbb{Q} \bar{w}_{1}(t)\right\|_{\mathcal{X}}+\left\|\mathbb{Q} C^{i} \mathbb{Q} \bar{w}_{1}(t)\right\|_{\mathcal{X}}+\left\|\partial_{t} \bar{w}_{1}(t)\right\|_{\mathcal{X}}\right\} \leq M_{T}
\end{aligned}
$$

Proof. Let us fix $0<T<\infty$. First we consider

$$
S \mathbb{Q} \bar{w}_{1}(t)=S\left(\mathbb{Q} C^{e} \mathbb{Q}\right)^{-1} \mathbb{Q} S \mathbb{P} \rho(t) .
$$

Due to (4.7), it is enough to analyze $t \rightarrow S^{2} \rho(t)$. By the discussion above, the assumptions are sufficient for the differentiation with respect to $\boldsymbol{x}$ to commute with the semigroup $(T(t))_{t \geq 0}$. Using Theorem 3.1

$$
\left\|S^{2} \rho(t)\right\|_{\mathcal{X}} \leq M_{1} \sum_{|\beta|=0}^{2}\left\|T(t) \partial_{\boldsymbol{x}_{\beta}}^{\beta} \stackrel{\circ}{v}\right\|_{\mathcal{X}_{2}} \leq M_{1}^{\prime}\|\stackrel{\circ}{v}\|_{\mathcal{X}_{220}}
$$

for some constant $M_{1}^{\prime}$, where for the multi-index $\beta=\left(\beta_{1}, \beta_{2}, \beta_{3}\right)$ we denoted $\partial_{\boldsymbol{x}_{\beta}}^{\beta}=\partial_{x_{1}}^{\beta_{1}} \partial_{x_{2}}^{\beta_{2}} \partial_{x_{3}}^{\beta_{3}}$. Next we consider

$$
\mathbb{Q} C^{i} \mathbb{Q} \bar{w}_{1}(t)=-\lambda^{-1} C^{i} S \rho(t) .
$$


Using (2.10), Theorem 3.1, and the commutativity of $C^{i}$ and $T(t)$, we obtain

$$
\begin{aligned}
\left\|\mathbb{Q} C^{i} \mathbb{Q} \bar{w}_{1}(t)\right\|_{\mathcal{X}} & \leq\|S \rho(t)\|_{\mathcal{D}\left(C^{i}\right)} \leq M_{2}^{\prime}\left\|\left(1+v^{-1}\right) S \rho(t)\right\|_{\mathcal{X}} \\
& \leq \sum_{|\beta|=0}^{1}\left\|v\left(1+v^{-1}\right) T(t) \partial_{\boldsymbol{x}_{\beta}}^{\beta} \stackrel{\circ}{v}\right\|_{\mathcal{X}} \\
& \leq \sum_{|\beta|=0}^{1}\left\|(1+v) T(t) \partial_{\boldsymbol{x}_{\beta}}^{\beta} \stackrel{\circ}{v}\right\|_{\mathcal{X}} \\
& \leq \sum_{|\beta|=0}^{1}\left\|T(t) \partial_{\boldsymbol{x}_{\beta}}^{\beta} \stackrel{\circ}{v}\right\|_{\mathcal{X}_{1}} \leq M_{2}^{\prime}\|\stackrel{\circ}{v}\|_{\mathcal{X}_{110}}
\end{aligned}
$$

uniformly on $[0, T]$. To estimate the next term we observe first that $\rho$ is differentiable in the norm of $\mathcal{X}_{2}$, thus we have

$$
\partial_{t} \bar{w}_{1}(t)=-\lambda^{-1} \mathbb{Q} S \mathbb{P} \partial_{t} \rho(t)=-\lambda^{-1} \mathbb{Q} S C^{i} \rho(t)
$$

Therefore

$$
\left\|\partial_{t} \bar{w}_{1}(t)\right\|_{\mathcal{X}} \leq M_{3}^{\prime} \sum_{|\beta|=0}^{1}\left\|T(t) C^{i} \partial_{\boldsymbol{x}_{\beta}}^{\beta} \stackrel{\circ}{v}\right\|_{\mathcal{X}_{1}} \leq M_{3}\|\stackrel{\circ}{v}\|_{\mathcal{X}_{111}} .
$$

The estimate of the first term in (4.20) follows easily as the assumptions of the lemma ensure that the function $t \rightarrow S \rho$ is continuous. Combining all the estimates we complete the proof of the lemma.

The regularity of the initial layer is dealt with in the next lemma.

Lemma 4.3. Let $\stackrel{\circ}{w}=\mathbb{Q} \stackrel{\circ}{f} \in \mathcal{X}_{111}$. Then there exists a positive constant $L$ such that for any $t \geq 0$

$$
\left\|S \mathbb{Q} \tilde{w}_{0}(t / \varepsilon)\right\|_{\mathcal{X}}+\left\|\mathbb{Q} C^{i} \mathbb{Q} \tilde{w}_{0}(t / \varepsilon)\right\|_{\mathcal{X}} \leq L e^{-\lambda_{\min } t / \varepsilon} .
$$

Proof. Let us consider first the term $\mathbb{Q} C^{i} \mathbb{Q} \tilde{w}_{0}(t / \varepsilon)$. Since $C_{-}^{i}$ is bounded, we see that we have to check only the behaviour of $C_{+}^{i} \tilde{w}_{0}(t / \varepsilon)$. Moreover, due to the explicit representation $\tilde{w}_{0}(t / \varepsilon)=e^{-\lambda t / \varepsilon} \stackrel{\circ}{w}$, the multiplication by $\alpha_{+}$commutes with the semigroup, hence the only troublesome part might be $K_{+}^{i} \tilde{w}_{0}(t / \varepsilon)$. We have however

$$
\begin{aligned}
\left\|K_{+}^{i} \tilde{w}_{0}(t / \varepsilon)\right\|_{X} & =4 \pi\left(\int_{S^{2}} \int_{0}^{\infty} \nu\left(\sqrt{v^{2}+1}\right) e^{-\sqrt{v^{2}+1} v^{-1} \lambda(v) t / \varepsilon} \stackrel{\circ}{w}(\boldsymbol{x}, v \boldsymbol{\omega}) d \boldsymbol{v}\right) \\
& \leq L^{\prime} e^{-\lambda_{\min } t / \varepsilon}\|\stackrel{\circ}{w}\|_{D\left(C^{i}\right)}
\end{aligned}
$$


for some constant $L^{\prime}$. The estimate in $\mathcal{X}$ is obtained by integrating the above inequality with respect to $\boldsymbol{x}$.

The estimate of $S \mathbb{Q} \tilde{w}_{0}(t / \varepsilon)$ is straightforward as $S$ commutes with the semigroup generated by $\mathbb{Q} C^{e} \mathbb{Q}$ and therefore the regularity of the initial data carries forward to the solution. The lemma is proved.

Combining all the considerations of this section we see that the following theorem is valid

Theorem 4.2. Assume that $\mathbb{P} \stackrel{\circ}{f} \in \mathcal{X}_{221}$ and $\mathbb{Q} \stackrel{\circ}{f} \in \mathcal{X}_{111}$. Let $f_{\varepsilon}$ be the solution of (4.1) with the initial data $\stackrel{\circ}{f}$, and $\rho$ be the solution to (4.11) with the initial value $\mathbb{P} \stackrel{\circ}{f}$. Then for each interval $[0, T], 0<T<+\infty$, there exists a constant $K$ depending only on the initial data, the coefficients of the equation and $T$ such that

$$
\left\|f_{\varepsilon}(t)-\rho(t)-e^{-\lambda t / \varepsilon} \mathbb{Q} \stackrel{\circ}{f}\right\|_{\mathcal{X}} \leq K \varepsilon
$$

uniformly on $[0, T]$.

Proof. For the proof we note that the assumptions on the initial data adopted here are stronger than that of Lemma 4.1 so that all the lemmas are available. Therefore all the steps of Subsection 4.1 are justified and by Lemmas 4.2 and 4.3 we have

$$
\begin{aligned}
\left\|e_{\varepsilon}(t)\right\|_{\mathcal{X}} \leq & \varepsilon\left\|\left(\mathbb{Q} C^{e} \mathbb{Q}\right)^{-1} \mathbb{Q} S \mathbb{P} \stackrel{o}{v}\right\|_{\mathcal{X}} \\
& +\varepsilon \int_{0}^{t}\left\|S \mathbb{Q} \bar{w}_{1}(s)+\mathbb{Q} C^{i} \mathbb{Q} \bar{w}_{1}(s)-\partial_{s} \bar{w}_{1}(s)\right\|_{\mathcal{X}} d s \\
& +\int_{0}^{t}\left\|S \mathbb{Q} \tilde{w}_{0}(s / \varepsilon)+\mathbb{Q} C^{i} \mathbb{Q} \tilde{w}_{0}(s / \varepsilon)\right\|_{\mathcal{X}} d s \\
\leq & \varepsilon M_{T}(1+T)+L \int_{0}^{t} e^{-\lambda_{\min } s / \varepsilon} d s \\
= & \varepsilon M_{T}(1+T)+\varepsilon L \int_{0}^{t / \varepsilon} e^{-\lambda_{\min } r} d r \leq K \varepsilon .
\end{aligned}
$$

The only difference now is that in (4.15) we had $e_{\varepsilon}=f_{\varepsilon}-\rho-\tilde{w}_{0}-\varepsilon \bar{w}_{1}$, whereas in (4.22) the last term is missing. However, the estimates of Lemma 4.2 can be carried also for $\bar{w}_{1}$ alone, showing that it is bounded on $[0, T]$. 
Since it is multiplied by $\varepsilon$, it can be moved to the right-hand side of the inequality without changing it. Hence the theorem is proved.

Acknowledgment. The author is very grateful to Professor G. Frosali who, having read an early version of this paper, pointed out several gaps and omissions.

\section{References}

[1] Abramovitz, M., Stegun, I. A., (eds.), Handbook of Mathematical Functions, Dover Publications, Inc., New York, 1965.

[2] Arendt, W., Resolvent positive operators, Proc. London Math. Soc. 54(3) (1987), 321-349.

[3] Banasiak, J., Mathematical properties of inelastic scattering models in kinetic theory, Math. Models Methods Appl. Sci. 10(2) (2000), 163-186.

[4] Banasiak, J., Diffusion approximation of an inelastic scattering model in linear kinetic theory, Adv. Math. Sci. Appl. 10(1) (2000), in print.

[5] Banasiak, J., On a diffusion-kinetic equation arising in extended kinetic theory, Math. Methods Appl. Sci. 23 (2000), in print.

[6] Banasiak, J., On the hydrodynamic limit of a linear kinetic equation with dominant elastic scattering, Atti Sem. Mat. Fis. Univ. Modena, accepted for publication.

[7] Banasiak, J., Frosali, G., Spiga, G., An interplay between elastic and inelastic scattering in models of extended kinetic theory and their hydrodynamic limts, in preparation.

[8] Banasiak, J., Frosali, G., Spiga, G., Asymptotic analysis for a particle transport equation with inelastic scattering in extended kinetic theory, Math. Models Methods Appl. Sci. 8(5) (1998), 851-874.

[9] Banasiak, J., Frosali, G., Spiga, G., Inelastic scattering models in transport theory and their small mean free path analysis, Math. Methods Appl. Sci. 23 (2000), 121145.

[10] Ben Abdallah, N., Degond, P., On hierarchy of macroscopic models for semiconductors, J. Math. Phys. 37(7) (1996), 3306-3333.

[11] Bobylev, A. V., Spiga, G., On a model transport equation with inelastic scattering, SIAM J. Appl. Math. 58, 1128-1137.

[12] Caraffini, G. L., Catalano, C.E., Spiga, G., On the small mean free path asymptotics of the transport equation with inelastic scattering, Riv. Mat. Univ. Parma (5) 1, (1998), 851-874.

[13] Demeio, L., Frosali, G., Diffusion approximations of kinetic equations with elastic and inelastic scattering: a comparison of different scalings, Sommari del IV Congresso Naz. Soc. Ital. di Matematica Industriale e Applicata SIMAI 98, Messina, 1-5 giugno 1998, Vol. 2, 316-319.

[14] Demeio, L., Frosali, G., Different scalings in the asymptotic analysis of kinetic equations with elastic and inelastic scattering, Universitá di Ancona, Dipartimento di Matematica, Rapporto N. 2 - Aprile 1999.

[15] Engel, K.-J., Nagel, R., One-parameter Semigroups for Linear Evolution Equations, Springer Verlag, Berlin, 1999.

[16] Frosali, G., Asymptotic analysis for a particle transport problem in a moving medium, IMA J. Appl. Math. 60 (1998), 167-185. 
[17] Garibotti, C. R., Spiga, G., Boltzmann equation for inelastic scattering, J. Phys. A 27 (1994), 2709-2717.

[18] Majorana, A., Space homogeneous solutions of the Boltzmann equation describing electron-phonon interaction in semiconductors, Transport Theory Statist. Phys. 20(4) (1991), 261-279.

[19] Markowich, P. A., Schmeiser, Ch., The drift-diffusion limit for electron-phonon interaction in semiconductors, Math. Models Methods Appl. Sci. 7(5) (1997), 707-729.

[20] Markowich, P. A., Ringhofer, Ch. A., Schmeiser, Ch., Semiconductor Equations, Springer Verlag, Wien, 1990.

[21] Mika, J. R., Banasiak, J., Singularly Perturbed Evolution Equations with Applications in Kinetic Theory, World Scientific, Singapore, 1995.

[22] Mokhtar-Kharroubi, M., Mathematical Topics in Neutron Transport Theory: New Aspects, World Scientific, Singapore, 1997.

\author{
JACEK BANASIAK \\ SCHOOL OF MATHEMATICAL \\ and Statistical Sciences \\ UNIVERSITY OF NATAL \\ DURBAN 4041 \\ South AFrica
}

